increasing propensity of some GPs to 'take on' and prescribe for patients who have failed with (or been failed by!) secondary services.

The Brighton and Hove Substance Misuse Service has now implemented the survey findings into new service standards and guidelines on the quality, frequency and content of future correspondence to GPs (Box 1). Despite an acceptable response rate, our results may not be generalisable elsewhere but our study might merit replication by other drug services.

\section{Conclusions}

Overall GPs view brief, timely and regular correspondence from substance misuse services as important. They placed particular importance on information with regard to medications being prescribed and required clear delineation as to what medications ought and ought not to be prescribed for patients by their GPs.

\section{Declaration of interest}

None.

\section{Acknowledgements}

We thank Ms Amanda Brookman, Clinical Librarian, Brighton and Sussex University Hospitals NHS Trust and our GP colleagues for their assistance.

\section{References}

BLAKEY, A., MORGAN J. \& ANDERSON, REYNOLDS, D. (1999) GPs' views on I. (1997) Communications between GPs discharge summaries and new patient and psychiatrists: the long and the assessment letters. Psychiatric Bulletin short of it. Psychiatric Bulletin, 21, 622-624.

DUNN, J. \& BURTON, S. (1999) GPS views on discharge summaries. Psychiatric Bulletin, 23, 355-357.

HICKMAN, M., HIGGINS, V., HOPE, V., et al (2004) Injecting drug use in Brighton, Liverpool and London: best estimates of prevalence and coverag of public health indicators. Journal of Epidemiology and Community Health, $\mathbf{5 8 , 7 6 6 - 7 7 1 . ~}$
SCOTT, A., MITCHELL, C. \& LOGANE. (2004) An audit of consultant physicians' reply letters for referral to clinics in a tertiary teaching hospital. Internal Medicine Journal, 34, 31-37.

WHITE,T. \& MARRIOTTS. (2004) Using evidence-based dissemination and mplementation strategies to improve routine communication between general practitioners and community mental health teams. Psychiatric Bulletin, 28, 8-11.

Devatha Radhae Shyam Specialist Registrar, Substance Misuse Service, South Downs Health NHS Trust, Brighton, *Hugh Williams Consultant Psychiatrist, Substance Misuse Service, South Downs Health NHS Trust, 26 Ditchling Road, Brighton BN1 4SF and Honorary Senior Lecturer, Department of Addictive Behaviour and Psychological Medicine, St George's Hospital Medical School, London SW17 ORE, e-mail: hugh.williams1@sussexpartnership.nhs.uk

\title{
Screening for smoking and substance misuse in pregnant women with mental illness
}

\author{
AIMS AND METHOD \\ Smoking and substance misuse \\ adversely affect the outcome of \\ pregnancy and psychiatric patients \\ are known to smoke more than other \\ patients. Data collected at the time of \\ routine antenatal booking were ana- \\ lysed to investigate whether preg- \\ nant women with mental health \\ problems smoke more than other \\ pregnant women.
}

\author{
RESULTS \\ Data were collected from 156 women. \\ Those with a psychiatric diagnosis \\ $(n=76)$ were significantly more likely \\ to smoke $(P<0.001)$. Associations \\ were also found with illicit drug use \\ and previous termination of preg- \\ nancy. The most common psychiatric \\ diagnosis was depression (62\%). A \\ diagnosis of schizophrenia was not \\ recorded for any of the women.
}

\author{
CLINICAL IMPLICATIONS \\ The strong association between \\ smoking and psychiatric diagnosis \\ results in an increased risk of obste- \\ tric complications in psychiatric \\ patients. Anti-smoking interventions \\ might be delivered by adequately \\ trained midwives and opportunisti- \\ cally during contact with mental \\ health professionals.
}

Smoking and substance misuse during pregnancy are known to adversely affect foetal outcome by a reduction in birth weight associated with intra-uterine growth retardation, preterm birth and an increased rate of perinatal death (Bennedsen, 1998). It is also known that psychiatric patients smoke more than other patients or the general public (McCreadie, 2002) and that women with schizophrenia have an increased risk of obstetric and perinatal complications (Sacker et al, 1996; Bennedsen, 1998; Howard et al, 2003). This increased risk has not been definitively accounted for; it is not known whether lifestyle differences such as smoking and substance misuse are explanatory, whether psychotropic medication is the causal factor or whether it is a result of genetic factors. There is only limited evidence supporting the assertion that pregnant women with mental illness are more likely to smoke and misuse substances than other pregnant women (Maughan et al, 2004), so we decided to investigate this at a routine antenatal clinic. 


\section{Method}

Women were identified from computer records as having booked their pregnancies during the period January 2001 to December 2002. Women with a psychiatric history according to the computer records were matched for age

\section{Table 1. Psychiatric diagnoses $(n=76)$}

Psychiatric diagnosis

Depression

Bipolar affective disorder

Schizophrenia

Schizoaffective disorder

Postnatal depression

Substance misuse

Eating disorder

Anxiety disorder

Other psychotic illness

Puerperal psychosis with a control group who had no psychiatric history. Information recorded included demographic data, obstetric and psychiatric history, and smoking and alcohol intake. Marital status was obtained from the booking record, but this did not specify whether unmarried partners were cohabiting. We were therefore unable to include this variable owing to the inaccuracy of these data.

Data analysis was carried out using Stata version 6 for Windows; $P$ values are all two-tailed. A descriptive analysis was carried out.

\section{Results}

Booking data were recorded for 156 women (mean age 30.1 years, s.d.=6). Psychiatric diagnosis was recorded by midwives and is given in Table 1. The psychotropic medications taken by the women at the time of booking were selective serotonin reuptake inhibitors ( $n=11,14.4 \%)$, tricyclic antidepressants ( $n=3,4.0 \%)$, antipsychotic drugs

Table 2. Variables in pregnant women with and without a psychiatric diagnosis

\begin{tabular}{|c|c|c|c|c|}
\hline Variable & Psychiatric diagnosis & No psychiatric diagnosis & $\chi^{2}$ & $P$ \\
\hline \multicolumn{5}{|c|}{ Length of gestation at booking, weeks } \\
\hline $3-9$ & 6 & 4 & 5.04 & 0.17 \\
\hline $10-19$ & 59 & 62 & & \\
\hline $20-29$ & 6 & 10 & & \\
\hline $30-39$ & 6 & 1 & & \\
\hline African-Caribbean & 11 & 15 & & $0.29^{1}$ \\
\hline Asian & 2 & 5 & & \\
\hline White & 49 & 41 & & \\
\hline Other & 12 & 17 & & \\
\hline \multicolumn{5}{|l|}{ Smoking } \\
\hline \multicolumn{5}{|l|}{ Alcohol use } \\
\hline Current & 30 & 23 & & \\
\hline \multicolumn{5}{|l|}{ Illicit drug use } \\
\hline None & 60 & 72 & 8.05 & 0.05 \\
\hline Recreational/dependent/ex & 9 & 3 & & \\
\hline \multicolumn{5}{|l|}{ Previous termination } \\
\hline No & 50 & 63 & 9.66 & 0.05 \\
\hline Yes & 27 & 14 & & \\
\hline \multicolumn{5}{|l|}{ Miscarriage } \\
\hline No & 49 & 53 & 1.03 & 0.31 \\
\hline Yes & 27 & 22 & & \\
\hline Spontaneous vaginal delivery & 26 & 47 & 1.79 & 0.62 \\
\hline Assisted vaginal delivery & 5 & 8 & & \\
\hline Elective Caesarean section & 8 & 7 & & \\
\hline Emergency Caesarean section & 7 & 9 & & \\
\hline
\end{tabular}


( $n=2,2.6 \%)$, mood stabilisers $(n=3,4.0 \%)$ and others $(n=2,2.6 \%)$. None of the women was taking atypical antipsychotic medication.

original

papers

There was no significant difference in ethnicity between women with and without a psychiatric diagnosis. Weeks of gestation at the time of booking did not differ significantly, but more very late bookers had a diagnosis of mental illness (Table 2). Women with a psychiatric diagnosis were significantly more likely to be smokers $(P<0.001)$, although the smokers in each group did not differ significantly in the amount smoked (Kruskal-Wallis test $P=0.55$ ). The median number of cigarettes consumed per day was 10 (interquartile range $5-15)$

Women with a psychiatric diagnosis were not significantly more likely to drink alcohol, although a trend was observed $(P=0.17)$. The median number of units consumed per week was 3 (interquartile range $2-4$ ). Twelve women had a history of current or previous illicit drug use, and these were significantly more likely to have a psychiatric diagnosis $(P=0.05)$. Those with a psychiatric history were significantly more likely to have a history of previous termination of pregnancy ( $P=0.05$ ), although there was no difference in history of miscarriage, stillbirth or neonatal death. Mode of delivery was compared between the two groups but no significant difference was found.

\section{Discussion}

We demonstrated a strong association between psychiatric history and smoking in pregnant women. We also found a significant association of psychiatric history with misuse of illicit drugs, but the numbers were small. No association was demonstrated between late booking and psychiatric history, confirming a similar finding in a UK study of women with psychotic disorders (Howard et al, 2003). This may reflect the nature of healthcare delivery in the UK, which is integrated and should prevent late booking in women with psychotic disorders once they have presented to their general practitioner.

The pattern of psychiatric diagnoses identified at the time of booking does not represent the epidemiological pattern. The absence of any women with schizophrenia could be a result of altered fertility in this group (Howard et al, 2002), inaccurate recording of psychiatric diagnoses by a relatively undertrained group of health professionals, and the stigma of such diagnoses leading to their underreporting by pregnant women.

Appropriately trained midwives might be able to correctly differentiate women with a psychiatric diagnosis from those suffering milder forms of distress, which is common in pregnancy. Having identified women with a psychiatric diagnosis they would need to be aware of potential stigmatisation, which would be likely to reduce the uptake of any help offered.

Interventions for smoking cessation should be tailored to this vulnerable group: just recycling the package offered to other women smokers is unlikely to be effective. Smoking cessation programmes are known to be of limited efficacy in pregnancy (Coleman, 2004). Other issues such as illicit drug misuse, alcohol misuse, misuse or appropriate use of prescribed psychotropic medication, and psychological or socio-economic risk factors (poor housing, lack of childcare) which may be associated with smoking and drug use might require specific intervention. It is unlikely that midwives could provide all the help required, but because the midwife is often the person who has most engaged the women, particularly when there are child protection issues resulting in distrust of mental health and social services, they could act in a liaison capacity with support services provided by other health professionals. Joint clinics between obstetricians and psychiatrists might be another opportunity for liaison between the disciplines caring for these patients.

Patient education regarding the harmful effects of smoking during pregnancy will influence the uptake of any intervention. The message that some psychotropic substances such as prescribed medication can have benefits that outweigh the risks in pregnancy must be disseminated by obstetricians, midwives and all other services involved in antenatal care. Finally, the interventions need to be appropriate for the educational level and cognitive function of the service user.

\section{Limitations}

Some information was incomplete and could not be included in the statistical analysis (marital status gave no indication of whether unmarried partners were cohabitees and the only indications of socio-economic status were address and occupation). The methodology did not account for differences in midwives' knowledge of mental illness. The stigma associated with psychiatric diagnoses might influence self-reporting of psychiatric history, especially with its implications of impaired abilities to care for the expected infant. Similarly, women might underreport smoking and other behaviours that are commonly known to impact negatively on foetal development.

Uptake of antenatal care is known to be lower in women with a history of substance misuse and therefore it is likely that there is an underrepresentation of this group. Many of the case records listed could not be found at the time of data collection, and if these notes were of more complex cases with other hospital appointments, there is the possibility of sample bias.

All data were collected by a single researcher, making it impossible to validate the reliability of the information gleaned. However, they were recorded exactly as they appeared in the midwives' booking records, were recorded on a purpose-designed form and entered onto the computer by the same researcher, thus limiting the possibility of misinterpretation.

\section{Implications}

Specific interventions to reduce smoking by pregnant women with mental illness are warranted, and would be likely to improve outcomes of pregnancy in terms of birth 
weight, preterm birth and neonatal morbidity and mortality. In turn, these outcomes are likely to result in increased well-being of this vulnerable group of women. Such interventions may have to be delivered opportunistically and psychiatrists may be best placed to do so. Therefore there is an onus on each of us to consider the physical well-being of our patients during pregnancy and to offer advice and education regarding smoking and other drug use.

\section{Declaration of interest}

None.

\section{References}

COLEMAN,T. (2004) Special groups of smokers. BMJ, 328, 575-577.

HOWARD, L. M., KUMAR, C., LEESE, M., et al (2002) The general fertility rate in women with psychotic disorders. American Journal of Psychiatry, 159, 991-997

HOWARD, L. M., GOSS, C., LEESE, M., et al (2003) Medical outcome of pregnancy in women with psychotic disorders and their infants in the first year after birth. British Journal of Psychiatry, 182, 63-67.

MAUGHAN, B., TAYLOR, A., CASPI, A., et al (2004) Prenatal smoking and early childhood conduct problems: testing genetic and environmental explanations of the association. Archives of General Psychiatry, 61, $836-843$

McCREADIE, R. G. (2002) Use of drugs, alcohol and tobacco by people with schizophrenia: case-control study. British Journal of Psychiatry, 181 321-325.

SACKER, A., DONE, D. J. \& CROW, T. J. (1996) Obstetric complications in children born to parents with schizophrenia: a meta-analysis of case-control studies. Psychological Medicine, 26, 279-287.
*Nisha Shah Specialist Registrar, North Islington Drugs Service, 592 Holloway Road, London N7 6LB, e-mail: nisha.shah@candi.nhs.uk, Louise Howard Senior Lecturer inWomen's Mental Health, Health Services Research Department, Institute of Psychiatry, London SE5 8AF

\section{Visual acuity and reported eye problems among psychiatric in-patients}

\section{AIMS AND METHOD}

The aim of this study was to examine visual problems among patients admitted to an inner city acute mental health unit. We measured visual acuity using a Snellen chart. Patients were also asked about perceived eye problems and access to services.

\section{RESULTS}

Of 55 in-patients on five acute general adult wards at an inner city mental health unit over a 3-day period, 31 agreed to participate in the study. Twenty $(65 \%)$ had impaired visual acuity and $19(61 \%)$ had not been to an optician for 5 or more years. Seventeen patients (55\%) reported experiencing difficulty with their eyesight. The main problems reported were blurring of vision and periorbital pain. Of these 17 patients, $15(88 \%)$ had impaired visual acuity on Snellen testing. Half of those who had previously been prescribed

\begin{abstract}
glasses or contact lenses reported that they had been lost.
\end{abstract}

\section{CLINICAL IMPLICATIONS}

Visual impairment appears to be another area of physical health which is underrecognised and undertreated in people with severe mental health problems. Although there are numerous issues that must be addressed by mental health staff, patients should be asked about eye problems and supported in accessing opticians.
There is substantial evidence that people with severe mental illness have poorer physical health than the general population and increased mortality from natural causes (Harris \& Barraclough, 1998). Few studies have specifically examined eye problems in people with severe mental illness. Two studies, conducted outside the UK, detected high rates of eye disease and untreated visual acuity problems (Smith et al, 1997; Ungvari et al, 2002). Furthermore, despite well recognised ocular side-effects of psychotropic medications, there are few data on the frequency with which these medications routinely cause visual impairment in psychiatric patients (McCarty et al, 1999).

Many of the factors known to have a detrimental effect on the physical health of people with severe mental illness (diet, alcohol consumption, lack of exercise and smoking) also affect vision. Moreover, common conditions such as diabetes mellitus and ischaemic heart disease are more common in this group than in the general population - and people with severe mental illness are less likely to access appropriate services.

To identify the incidence of impaired visual acuity we conducted a cross-sectional descriptive study of psychiatric in-patients with severe mental illness and collected subjective information about eye problems and access to services.

\section{Method}

In-patients from five acute general adult wards at an inner city mental health unit were invited to participate in the study. Patients who were acutely intoxicated or 